\title{
Fatigue Behaviour of a Gold-Nickel Alloy
}

\section{INFLUENCE OF TESTING CONDITIONS ON THE CRACK GROWTH RATE}

\author{
Markus O. Speidel \\ BBC Brown Boveri Research Center, Baden, Switzerland
}

\begin{abstract}
The growth rates of fatigue cracks in massive plates of a gold-nickel alloy agree reasonably well with a quantitative prediction applicable to other metallic materials. This implies that fatigue crack growth rates in other gold-based alloys can also be predicted with a degree of confidence.
\end{abstract}

Fatigue is characterized by the initiation and growth of cracks in solids submitted to cyclic stresses. While literature pertaining to various forms of fatigue in common base metals and their alloys is abundant, very few articles have been devoted to high-cycle fatigue, thermal fatigue and corrosion fatigue in gold and its alloys. Those that have been published ( 1 to 4 ) describe the fatigue resistance of small and smooth specimens where the number of load cycles to initiate a fatigue crack alone determines the fatigue life. Nothing has yet been reported about the growth of fatigue cracks in gold alloys. The present article reports quantitative fatigue crack growth rates in massive specimens of a gold alloy.

\section{The Gold-Nickel Alloy}

The alloy chosen for the investigation of fatigue crack growth rates contained 95 per cent gold and 5 per cent nickel by weight. The material was received and used in the form of a rectangular plate $100 \times 60 \times 10$ $\mathrm{mm}$. The alloy was in the cold-rolled condition with a Vickers hardness of $1650 \mathrm{MN} / \mathrm{m}^{2}$, corresponding to a yield strength, $\sigma_{0.2}$, of $500 \mathrm{MN} / \mathrm{m}^{2}$ and an ultimate tensile strength of $560 \mathrm{MN} / \mathrm{m}^{2}$. Its modulus of elasticity $\mathrm{E}$ was measured to be $88 \mathrm{GN} / \mathrm{m}^{2}$.

This commercial alloy is used for a variety of applications where high strength and a clean metallic surface are important; for example, in electrical contacts and switches. The alloy was selected for this investigation on account of its high gold content together with high strength. The latter property is desirable for fracture mechanics studies because it ensures predominantly plane strain conditions (5).

\section{Experimental; Fracture Mechanics}

The growth rate of fatigue craclss was measured using linear elastic fracture mechanics techniques. The increase in crack length per load cycle, $\Delta \mathrm{a} / \Delta \mathrm{N}$, was determined using the specimen type shown in Figure 1. The progress of the fatigue crack length, $\Delta \mathrm{a}$, was measured on both sides of the specimen, using a calibrated low-power microscope. The corresponding cyclic stress intensity range, $\Delta \mathrm{K}$, was determined according to the K-calibration shown in the equation of Figure $1,(6)$. To obtain $\Delta \mathrm{K}$ from this equation, the load excursion $P$ was measured on the load cell of the hydraulic fatigue machine and inserted into the equation, together with the actual crack length a and the specimen dimensions $\mathbf{H}$ and $\mathbf{B}$. The test piece had to exceed certain minimum dimensions to prevent buckling and gross plastic deformation and to ensure plane strain conditions. Predominantly plane strain conditions are achieved if the smallest dimension, $\mathbf{B}$, exceeds $2.5\left(\mathrm{~K} / \sigma_{0,2}\right)^{2}(6,7)$. With specimen thickness $\mathrm{B}=10^{-2} \mathrm{~m}$ and yield strength $\sigma_{0.2}=500 \mathrm{MN} / \mathrm{m}^{2}$, plane strain conditions prevail at stress intensities up to $\mathrm{K}=32 \mathrm{MNm}^{-3 / 2}$.

Figure 2 shows a photograph of the test piece used, together with a well known gold coin. It will be noted that the fatigue specimen was massive; it weighed more than one kilogram.

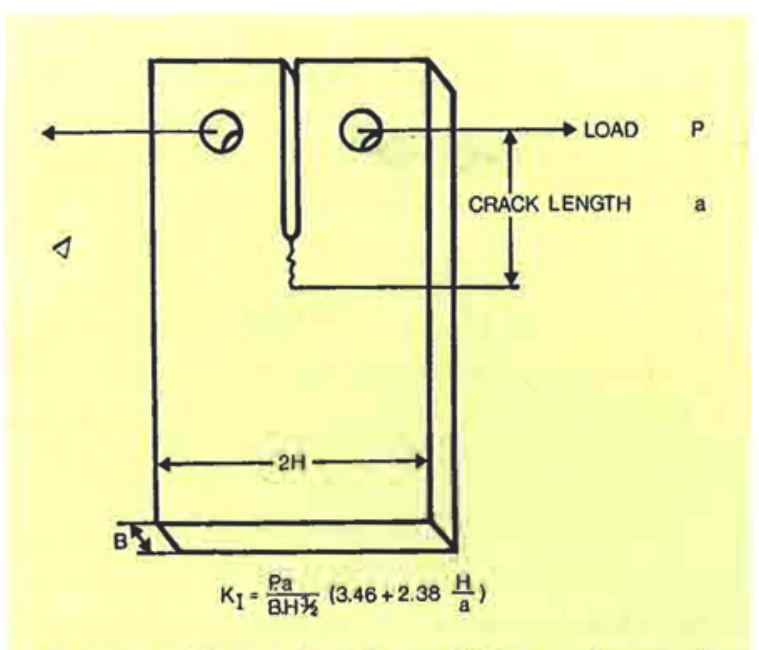

Fig. 1 Double cantilever beam (DCB) specimen and stress intensity calibration used in this study to measure the fatigue crack growth rate as a function of the cyclic stress intensity range $\Delta K$ 

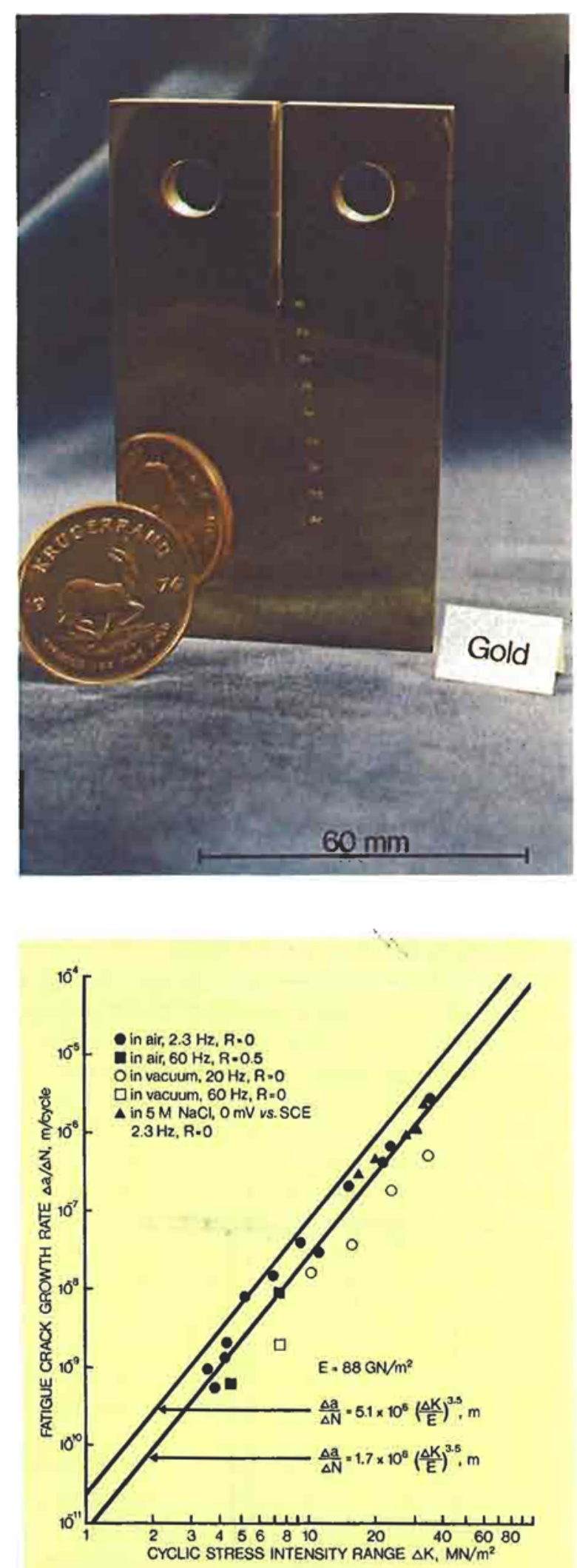

Fig. 3 Effect of the cyclic sinusoidal stress intensity range, the ratio $\mathbf{R}$ of minimum to maximum load and the loading frequency on the growth rate of fatigue cracks in a massive specimen of 95 gold $/ 5$ nickel weight per cent alloy exposed to various environments at $23^{\circ} \mathrm{C}$
Fig. 2 A massive specimen of 95 gold/5 nickel weight per cent alloy was used by the author for fatigue crack growth rate determination. It is shown here at the conclusion of the testing programme and the crack initiated at the tip of the machined notel extends over more than $60 \mathrm{~mm}$

The same specimen was used to measure the modulus of elasticity of the 95 gold $/ 5$ nickel weight per cent alloy. This was done using a method specifically developed for pre-cracked fracture mechanics specimens, which is described elsewhere (8). The measured modulus, $\mathrm{E}=88 \mathrm{GN} / \mathrm{m}^{2}$, agrees well with values expected for similar gold alloys. In the course of the fatigue tests, the frequency of the load cycling was varied from 2.3 to $60 \mathrm{~Hz}$. The ratio $\mathrm{R}$ of the minimum to the maximum load was varied from 0 to 0.5 .

\section{Test Results; Fatigue Crack Growth Rates}

The effect of the cyclic stress intensity range, $\Delta \mathrm{K}$, on the growth rates of fatigue cracks in the gold-nickel alloy studied here is shown in Figure 3 for several environments. Fatigue crack growth rates in vacuum are on average about one third of the crack growth rates measured in air or in $5 \mathrm{M}$ sodium chloride aqueous solution. Of the mechanical test variables, the loading frequency and the ratio $R$ of minimum to maximum load have no measurable influence, while in contrast, $\Delta \mathrm{K}$ has a dominant influence on the fatigue crack growth rate. Two straight lines and their mathematical expression are also shown in Figure 3. The majority of the fatigue crack growth rates measured in air and in salt solution fall between these two lines, while data measured in vacuum fall short of the lower line. The two lines and the corresponding equations represent general predictions of fatigue crack growth rates in air and in vacuum for all metals and alloys for which the modulus of elasticity is known (9). It is apparent that these predictions are an upper limit of the measured fatigue crack growth rates in the gold-nickel alloy. Thus, fatigue life time predictions based on an integration of the equations shown in Figure 3 would be conservative. The actual data show fatigue crack growth in gold $/ 5$ per cent nickel to be slower by a factor two to three. This is as good an agreement as one might expect from a method of prediction in which the only property of a material taken into account is its modulus of elasticity. The effect of stress intensity on the growth rate of fatigue cracks is well predicted by the exponent 3.5 in the equations of Figure 3.

Since the general modulus correlation (9) predicts the fatigue crack growth rates in the 95 gold $/ 5$ nickel per cent alloy quite satisfactorily, it may reasonably 
be expected that fatigue crack growth rates in other gold alloys can also be derived from the equations shown in Figure 3, using the appropriate values of $\mathrm{E}$. For example, the fatigue crack growth rates in pure gold may be predicted by inserting the appropriate modulus, $\mathrm{E}=81 \mathrm{GN} / \mathrm{m}^{2}$, in these equations.

\section{Corrosion Fatigue Crack Growth}

The fatigue crack growth rate data plotted in Figure 3 show no measurable difference for air and sodium chloride solution as test environments; only the crack growth rates in vacuum are lower. However, Figure 4 illustrates that the electrode potential of the gold alloy in the salt solution has a marked effect on fatigue behaviour. Also shown in Figure 4, is the effect of the electrode potential on the current density in a corrosion cell where the alloy is the working electrode.

The effect of potential on the growth rate of fatigue cracks in the gold-nickel alloy is analogous to those which have been observed with aluminium alloys and with silver $(10,11)$. The corrosion fatigue crack growth is markedly reduced towards more negative potentials (cathodic protection), but even so, crack growth is still faster than in vacuum. Towards more positive potentials, from -300 to $+600 \mathrm{mV}$ vs. a standard hydrogen electrode (SHE), the corrosion fatigue crack growth rates are uniformly high. At still more positive potentials, a reproducible minimum of the corrosion fatigue crack growth rate is observed over a narrow potential range near the open circuit potential.

Among the presently discussed theories for corrosion fatigue crack growth, two are particularly prominent: one attributes the effects of environments on the phenomenon to preferential anodic dissolution at the crack tip, the other attributes it to preferential adsorption of specific ions at the crack tip in specific potential ranges. An extensive study is currently under way to investigate the effects of the electrode potential on corrosion fatigue crack growth in many metals. Systematic comparisons of the behaviour of base and noble metals will hopefully shed light on the theories for corrosion fatigue crack growth. In this respect, the sharp minimum of the corrosion fatigue crack growth rate in the gold-nickel alloy at $+750 \mathrm{mV}$ vs. SHE is a new and unexpected phenomenon which is not yet understood.

\section{Summary and Conclusions}

The growth rates of fatigue cracks in the gold alloy with 5 per cent nickel by weight, follow approximately the equation:

$$
\Delta \mathrm{a} / \Delta \mathrm{N}=1.7 \times 10^{6}(\Delta \mathrm{K} / \mathrm{E})^{3.5}
$$

where $-a / \Delta N$ is the fatigue crack increment per load cycle, $K$ is the cyclic stress intensity range and $E$ is the modulus of elasticity (here $88 \mathrm{GN} / \mathrm{m}^{2}$ ). Fatigue

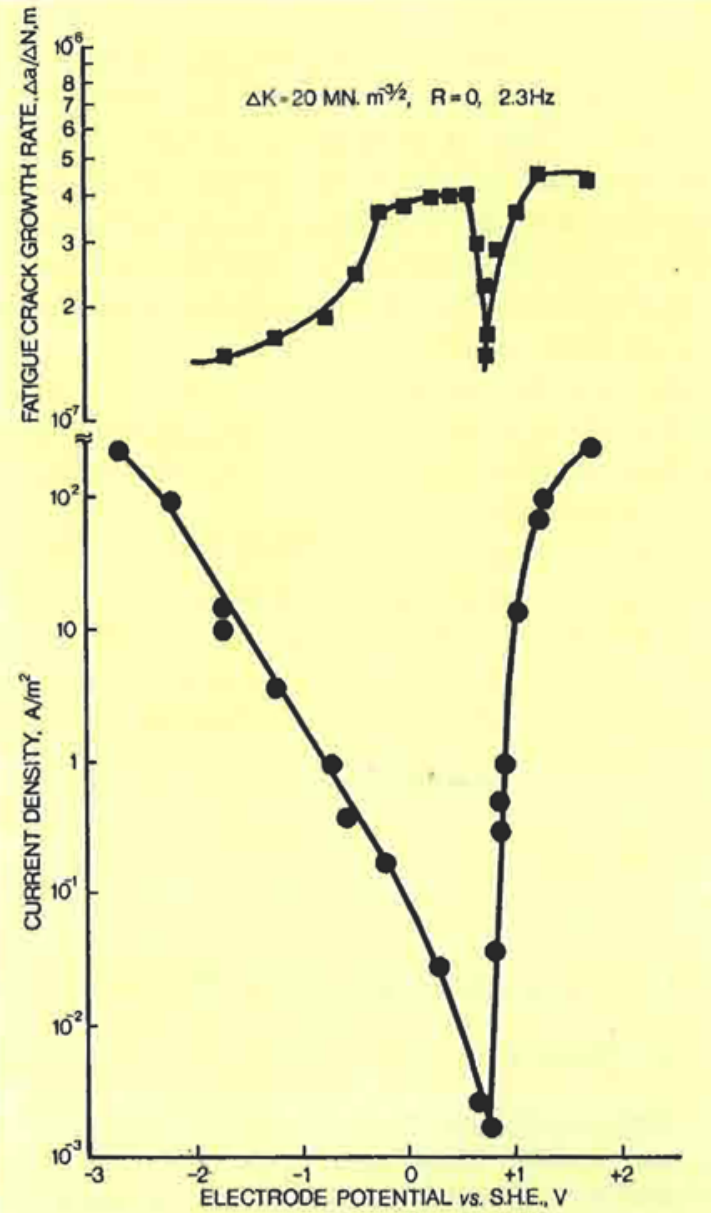

Fig. 4 The application of an electrical potential (expressed here vs. a standard hydrogen electrode) has a marked effect on the growth rate of fatigue cracks in a specimen of 95 gold $/ 5$ nickel weight per cent alloy exposed to a 5 molar aqueous solution of sodium chloride (pH 6) at $23^{\circ} \mathrm{C}$. The sharp minimum recorded in the fatigue crack growth rate at $+750 \mathrm{mV}$ vs. SHE is reproducible but still has to be explained

crack growth rates in air and $5 \mathrm{M}$ sodium chloride aqueous solution are up to three times faster, and fatigue crack growth rates in vacuum are about three times slower than equation (1) indicates.

Since equation (1) is generally applicable as an approximation to the fatigue crack growth rates of many metals and alloys, it may be used for different gold alloys as well. The effects of alloy composition and testing temperature are taken into account in the fatigue crack growth rate prediction according to equation (1) through the modulus of elasticity $\mathrm{E}$. It has also been shown $(9,11,12,13)$ that equation (1) accounts for the behaviour of many alloys quite accurately, whether they are single- or multi-phase, and whether they are solid solution- or precipitationhardened. This is particularly true for the intermediate fatigue crack growth rates and the intermediate stress intensity ranges for which data 
have been gathered in Figure 3. To be on the safe side, general predictions based on equation (1) should be limited to the $\Delta \mathrm{a} / \Delta \mathrm{N}$ and $\Delta \mathrm{K}$ ranges shown in Figure 3. This is because some effects of the microstructure on the fatigue behaviour of metals have been observed at extremely low and at very high $\Delta \mathrm{K}$ 's, where respectively the threshold values for fatigue crack growth, $\Delta \mathrm{K}_{0}$, or the fracture toughness, $\mathrm{K}_{\mathrm{Ic}}$, are approached (12).

The electrode potential has a marked effect on the growth rate of fatigue cracks in the gold alloy when exposed to sodium chloride solution. The reduction of the crack growth rates at negative potentials and their acceleration between $-300 \mathrm{mV}$ and $+600 \mathrm{mV}$ vs. SHE are closely analogous to earlier observations with aluminium alloys and pure silver. It is hoped that further comparisons and studies of the kind presented here will clarify the mechanistic aspects of corrosion fatigue crack growth.

\section{References}

1 F. A. Peyton, 7. Am. Dent. Assoc., 1934, 21, 394-415

2 J. L. Dais and F. L. Howland, IEEE Trans. Compon. Hybrids Manuf. Technol, 1978, CHMT-1, 158-166

3 N. E. Frost, K. J. Marsh and L. P. Pool, 'Metal Fatigue', Ox ford Engineering Series, Clarendon Press, Oxford, 1974

4 L. Graf and W. Richter, Metall, 1977, 32, 1332-1338

5 W. F. Brown and J. E. Strawley, 'Plain Strain Crack Toughness Testing of High Strength Metallic Materials', ASTM Special Technical Report 410, Am. Soc. Test. Mater., Philadelphia, U.S.A., 1969

6 J. E. Strawley and B. Cross, Mater. Res, Stand., 1967, 7, 155

7 ASTM Standard E 399-74

8 M. O. Speidel, f. Mater. Technol, 1976, 7, 364-367

$9 \mathrm{M}$. O. Speidel, in 'High-Temperature Materials in Gas Turbines', Elsevier, Amsterdam, 1974

10 M. O. Speidel, M. J. Blackburn, T. R. Beck and J. A. Feeney, in 'NACE-2', Natl. Assoc. Corros. Eng., Houston, U.S.A., 1972, pp. 324-345

11 M. O. Speidel, in 'Handbook of Stress Corrosion Cracking and Corrosion Fatigue', to be published

12 M. O. Speidel and R. B. Scarlin, in 'Gefüge und Bruch', Materialkundlich-Techn. Reihe, Vol. 3, Borntraeger, Berlin, 1977, pp. 163-189

$13 \mathrm{M}$. O. Speidel, in 'Stress Corrosion Cracking and Hydrogen Embrittlement of Iron Base Alloys', NACE-5, Natl. Assoc. Corros. Eng., Houston, U.S.A., 1977

\section{Generation of Power by Nuclear Fusion}

\section{A PROCESS WHICH MAY INVOLVE USE OF GOLD}

The basic objective in the concentrated efforts which are currently being made to harness energy from nuclear fusion reactions and use it for generation of electric power can be stated simply. It is to establish the necessary technology for heating and compressing fusionable fuels to the point where thermonuclear reactions occur, releasing more energy than is consumed in the process. For example, if the fuel is a mixture of the hydrogen isotopes deuterium and tritium, energy may be released as follows:

$$
\mathrm{l}^{2} \mathrm{D}+1{ }^{3} \mathrm{~T} \longrightarrow 2{ }^{4} \mathrm{He}+\mathrm{n}+17.6 \mathrm{MeV}
$$

One approach to the problem is to enclose the fuel in small pellets which are made to implode by exposure either to powerful laser beams or to beams of electrons or ions generated by very high current, very high voltage pulses of electricity. In this manner the fuel is raised to the extreme temperatures and densities which are necessary to initiate the desired fusion reaction. The work is being pursued in two centres, at the Sandia Laboratories in Albuquerque, New Mexico, and at the I.V. Kurchatov Institute of Atomic Energy in Moscow.

The design of the fuel pellets is of major importance and in investigations which have been reported upon (G. Yonas, Sci. Am., I979, 239, (5), 40-5I), they have been small concentric spheres. The innermost sphere has contained the hydrogen isotope fuel and it has been surrounded (see A. Mayer and D. S. Catlett, Plat. Surf. Finish., I978, 65, (3), 42-46) by a 'pusher' sphere of pure gold, coated on the outside by an explosive 'ablator' material. When the pellets are exposed to laser, electron or ion beams, the energy of the latter is absorbed in the ablator layer, which explodes. The intermediate pusher shell then serves to compress and heat the fuel symmetrically. The latter is forced to the centre of the pellet where the temperature and pressure conditions developed are such as to ignite the fusion reaction in the fuel. In experiments at the Sandia Laboratories, loss of energy from the imploding fuel to the pusher shell has been reduced by the production of a trapped magnetic field within the pellet by discharge of a high current pulse of electric energy through a filament embedded in the pellet. In this way the final temperature attained by the fuel has been raised and the extent of the fusion reaction (as measured by the neutron yield) increased significantly.

Gold is apparently an ideal pusher material for bringing the atoms of fuel together. Not only does it prevent penetration of the pellet by the pulsed laser, electron or ion beam and so assist in confining absorption of the energy of the beam to the ablator layer, but when this ablator layer explodes, it shields the fuel in the centre of the pellet from the radiant energy associated with the explosion. Heating up and expansion of the fuel before it implodes are therefore reduced to a minimum.

It has been calculated that a laser fusion power plant of $1000 \mathrm{MW}$ capacity could consume about one million fuel pellets per day, and that these would contain about $3 \mathrm{~kg}$ of gold. Although this gold would be recoverable, an inventory of about $960 \mathrm{~kg}$ of the metal would be required for each $1000 \mathrm{MW}$ production capacity. Current indications are that an electron beam fusion power plant would be more efficient than a laser beam plant, but that it would call for considerably greater gold inventories. 